Dennison, L., Moss-Morris, R., Silber, E., Galea, I., \& Chalder, T. (2010). Cognitive and behavioural correlates of different domains of psychological adjustment in early stage Multiple Sclerosis. Journal of Psychosomatic Research, 69(4), 353-361.

\title{
Cognitive and behavioural correlates of different domains of psychological adjustment in early stage Multiple Sclerosis
}

\author{
Laura Dennison, Rona Moss-Morris, Eli Silber, Ian Galea and \\ Trudie Chalder
}

\begin{abstract}
Objective: This study investigated a cognitive-behavioural model of adjustment to Multiple Sclerosis (MS). It aimed to determine the contribution of cognitions and behaviours to the explanation of two distinct adjustment outcomes above and beyond measures of MS severity. Illness-related functional impairment was anticipated to be most strongly related to unhelpful thoughts and behaviours that were specific to MS and the experience of symptoms. Psychological distress was hypothesised to be most strongly related to more general unhelpful cognitions about the self and emotions.
\end{abstract}

Methods: 94 people with MS completed questionnaires. Correlations and hierarchical multiple regressions determined the relative contribution of illness severity, cognitions, and behaviours to the prediction of psychological distress and functional impairment.

Results: Illness-related functional impairment was related to disease severity, progressive vs. relapsing-remitting disease and unhelpful illness perceptions and cognitive and behavioural responses to symptoms. Illness severity factors accounted for a significant $23.7 \%$ of the variance in functional impairment $(p<.001)$. Cognitive and behavioural variables explained a further $22.6 \%$ of variance $(p<.001)$ with behavioural responses to symptoms emerging as the strongest predictor. The correlates of distress were unhelpful beliefs about the self, unhelpful beliefs about emotions, acceptance, and unhelpful cognitive responses to symptoms and illness perceptions. Illness severity factors explained only $2.2 \%$ of the variance in distress $(p>.05)$ while cognitive and behavioural variables accounted for $37.1 \%(p<.001)$. Unhelpful beliefs about the self were the strongest predictor.

Conclusion: Longitudinal and experimental research is required to investigate potential causal relationships. However, the cognitions and behaviours identified as important for adjustment are potentially modifiable, so may be useful to address within interventions for adjustment to MS.

Keywords: Multiple Sclerosis, Adjustment, Distress, Impairment, Cognitive Behavioural 
Multiple Sclerosis (MS) is a neurological disease which is thought to affect more than 2.5 million people worldwide (1). MS symptoms vary enormously between people with MS (PwMS) but can include spasticity, disturbances to strength, balance, sensation and vision, bowel and bladder disturbances, sexual dysfunction, cognitive impairment, pain and fatigue. Around $85 \%$ of patients present with a relapsingremitting form of MS. However for the majority, the disease progresses over time and impairment increases (2). Although there is no cure for MS, the available diseasemodifying drugs are somewhat successful in reducing the severity and frequency of relapses and disease progression (3;4).

Individuals with MS are faced with uncertainty about the future, unpleasant and unpredictable symptoms, difficult treatment regimes and drug side effects. MS can have profound consequences including the disruption of life goals, employment, income, relationships, social and leisure activities and activities of daily living. This may be particularly significant because for the majority of PwMS the disease begins in young adulthood, a period often important for career development and starting families. Therefore it is not surprising that many patients encounter difficulties with psychosocial adjustment. Research demonstrates elevated levels of depression and psychological distress $(5 ; 6)$ anxiety (7), relationship and social dysfunction $(8 ; 9)$ and reduced quality of life $(6 ; 10)$ in PwMS.

Illness-related factors can influence levels of adjustment (e.g. 11;12). Such factors include MS type, length of illness and remission status. MS severity also appears to be important; this is most commonly measured by the Expanded Disability Status Scale scale (EDSS; 13) which assesses gait disturbance and other symptoms including cognitive impairment, visual disturbances and bladder dysfunction. However, illnessrelated factors are not consistently associated with adjustment outcomes and only predict modest amounts of the variance. In a recent systematic review of the psychological literature in MS we showed that a range of cognitive and behavioural factors are important in predicting and explaining individual differences in adjustment (14). Importantly, it may be possible to address these factors in psychological interventions in order to improve adjustment in PwMS. As a result of the review we suggested a cognitive-behavioural model of adjustment to MS. According to the model, MS development, diagnosis or progression are conceptualised as critical events which disrupt emotional equilibrium and quality of life, at least in the short term (13). Whether a person continues to be distressed and perceive their quality of life as poor in the longer term is influenced by a range of variables. These include individuals' cognitive and behavioural responses to the critical event, as well as social and environmental factors. Our review identified factors for which existing research had clearly established links to adjustment (e.g. coping strategies, perceived social support). However, we also suggested a number of cognitive and behavioural variables which warrant further investigation (14).

Our review suggested that variables derived from health psychology frameworks, such as illness representations (15) and how people interpret and respond to symptoms (16) may be important for adjustment. There have however, been few studies of these factors thus far. The role of acceptance in MS also requires more research. Only two studies identified in the review examined acceptance. Both 
explored its relationship to marital adjustment and the findings were inconsistent $(17 ; 18)$. The paucity of research into acceptance is surprising given the enduring belief held by many patients and health professionals that developing an acceptance of the disease is critical for adjustment (19).

The review also indicated that variables derived from cognitive models of psychopathology may be important for understanding and predicting depression in PwMS. These include cognitive biases towards negative information and attributions and unhelpful or negative beliefs about oneself, the world and the future. However, only a handful of studies have been conducted so far (20-24) and these factors have not been studied in relation to broader adjustment outcomes such as social and role functioning and quality of life. Another variable deemed worthy of exploration is people's beliefs about experiencing and expressing negative emotions. Although no existing quantitative studies have explored this area, our recent qualitative study suggested beliefs about emotions are important (25). Our participants described how strong negative emotions are almost inevitable in response to MS-related challenges such as diagnosis and relapse. Participants also expressed feeling that ongoing negative emotions should not be tolerated and that demonstrating positivity and 'putting on a brave face' is desirable. Such beliefs about the unacceptability of negative emotions have been noted in populations with other mental and physical health problems (26-29) and are thought to play a role in the development and maintenance of clinical problems (30).

The current study sought to extend promising areas of research highlighted above. We aimed to understand the types of variables which are related to adjustment in order to pinpoint factors which could be targeted by interventions which seek to improve adjustment outcomes for PwMS. Adjustment is multifaceted, comprising various outcomes such as psychological distress, functional impairment and quality of life. Therefore, this study explored how different factors might contribute to different forms of adjustment. We examined functional impairment (the impact of MS on ability to perform key roles such as work and social activities) and psychological distress. These outcomes capture two key aspects of adjustment, and although potentially related, these are in fact distinct dimensions.

This study also addressed some important methodological limitations of previous studies. Most existing studies fail to measure or account for the influence of illnessrelated factors such as MS type and disability status. They cannot therefore conclude that the psychological factors which explain variance in adjustment outcomes are not simply a response to more severe and advanced disease. This study examined the influence of cognitive and behavioural variables over and above MS type and severity factors. It also addressed sampling problems inherent in existing research. Many studies do not distinguish between patients at different points in their disease trajectories where adjustment issues may differ. Very few studies have examined adjustment in people relatively early on in their disease course despite research suggesting that distress is apparent early on and that patients desire psychological support at this stage (6;25). We, therefore, specifically sampled patients early on in their disease trajectory. Furthermore, previous studies have typically drawn participants from voluntary patient organisations who represent only a percentage of all PwMS. Those who join such support groups may have different ways of dealing 
with the illness than those who do not. In order to sample a broader group of people with MS this study recruited through hospital and community based MS services.

We predicted that cognitive and behavioural factors would explain significant variance in distress and functional impairment in early stage MS over and above illness severity variables. Specifically we hypothesised that MS-specific cognitions and behaviours would be particularly important in determining functional impairment. Thus, people who think very negatively about their MS and symptoms or who have unhelpful behaviours linked to symptoms would perceive that MS imposes more limits on life roles. We also expected that unhelpful cognitions which are not MSspecific would be most important for determining distress. Thus unhelpful beliefs about the self and unhelpful beliefs about emotions would be associated with distress. MS-specific cognitions and responses would also be relevant; lack of acceptance, negative illness perceptions, and unhelpful behavioural responses to symptoms would be related to higher distress.

\section{Method}

This cross-sectional study was nested within a randomised controlled trial of psychological interventions for adjustment to early stage MS (31). The data presented here is from the baseline questionnaires completed prior to randomisation to a treatment group. The study was approved by Thames Valley Multi-centre Research Ethics Committee (07/MRE12/6).

\section{Participants}

Participants were recruited from two National Health MS Services: the Wessex Neurological Centre in Southampton and King's College Hospital in South London. During consultations, nurse specialists and neurologists informed patients who met eligibility criteria about the study. Potential participants then had the opportunity to discuss the study with the trial co-ordinator before deciding whether to take part. Eligibility was confirmed by a telephone screening interview after written consent was given.

To be eligible for inclusion in the trial patients had to have a definite diagnosis of MS within the last ten years. They had to be able to walk a distance of at least $20 \mathrm{~m}$ with bilateral support (EDSS of 6.5 or less (13)). Patients with other serious health problems, severe mental health problems (e.g. psychosis), or severe cognitive impairment were excluded. Participants did not have to be currently experiencing adjustment difficulties.

161 patients contacted the researchers to express interest and 112 (69.6\%) consented to participate. Eight of these were not eligible at screening, six changed their mind and four were not contactable. This resulted in a sample of 94 participants who completed a baseline questionnaire assessment by post. 


\section{Measures}

\section{Demographic and illness measures}

Patients completed a demographic data questionnaire and self-reported information about their MS. They also underwent the Telephone Interview for Cognitive Status Modified (TICS-M; 32) to screen for potential cognitive impairment which would make participation in the study inappropriate (a score of $<20$ ). Participants also completed a self-report EDSS which has been shown to correlate well with physician rated scores (33). EDSS questionnaires were scored by LD (a health psychology researcher) and later co-rated by IG (a neurologist experienced in EDSS assessment) in order to ensure reliability of scoring. Kappas for all items were $>.70$ (substantial agreement), with most $>.80$ (excellent agreement).

\section{Adjustment outcomes}

The Work and Social Adjustment Scale (WSAS; 34) measures how much an identified illness (in this case MS) impairs the person's work, home management, social and leisure activities and relationships. Higher scores indicate greater functional impairment. The WSAS has excellent psychometric properties and has been previously used in MS research (35). Cronbach's alpha in this sample was excellent (.84).

The General Health Questionnaire-12 (GHQ-12; 36) measures psychological distress in people in community and medical settings. Higher scores indicate greater distress. The measure has good psychometric properties and a recent study which found it to be the most treatment-responsive measure of distress in MS (37). A Cronbach's alpha of .91 indicated excellent internal reliability.

\section{Potential predictors of adjustment}

The Psychological Vulnerability Scale (PVS; 38) measures unhelpful beliefs about the self. It assesses maladaptive cognitive responses which are proposed to promote unhelpful responses to stressors (e.g. perfectionism, need for approval). High scores indicate more maladaptive thinking. Good internal reliability was demonstrated in this sample (.756).

The Beliefs about Emotions Scale (30) measures unhelpful beliefs about emotions. It measures the extent to which the person holds beliefs that it is intolerable and unacceptable to experience negative emotions, express emotion or weakness to others, and that negative emotions should be carefully controlled. High scores indicate more unhelpful beliefs about emotions. An alpha of .84 in this study indicated excellent reliability.

The Acceptance of Chronic Health Conditions scale (ACHC; 39) assesses acceptance of, and adjustment to, change in one's life due to a chronic health condition. High scores indicate greater acceptance of MS. Cronbach's alpha in this sample was excellent (.83).

The Brief Illness Perception Questionnaire (BIPQ; 40) assesses cognitive and emotional illness representations (consequences, timeline, personal control, treatment 
control, illness identity, concern, coherence and emotional response). High scores reflect negative perceptions of aspects of the individual's MS. Internal reliability for the BIPQ total score was poor (.57) so individual item scores were used in analyses. Furthermore, two BIPQ items were somewhat confounded with the outcomes (the 'emotional responses' item overlapped with the GHQ and the 'consequences' item overlapped with the WSAS) and were therefore omitted from the applicable analyses.

The Cognitive and Behavioural Responses to Symptoms Questionnaire (CBRSQ; 41) is a newly-devised scale which assesses patients' cognitive and behavioural responses to the experience of symptoms. This study used a 34 item version. The five subscales dealing with cognitive responses are symptom focusing (e.g. "I think a great deal about my symptoms”), catastrophising (e.g. "I will never feel right again”), damaging beliefs (e.g. "Symptoms are a signal that I am damaging myself "), fear avoidance, (e.g. "I should avoid exercise when I have symptoms ") and embarrassment avoidance ( e.g. "The embarrassing nature of my symptoms prevents me from doing things"). The two behavioural subscales measure all-or-nothing responses (e.g. "I find myself rushing to get things done before I crash") and avoidance/resting (e.g. "I stay in bed to control my symptoms"). High scores indicate more unhelpful responses. Alpha was .84 for both the cognitive and behavioural responses scales.

\section{Data analysis}

Data was screened and checked against the assumptions of regression analysis. The BIPQ timeline item was extremely negatively skewed so this was dichotomised into those who believed their MS would last forever (the vast majority) and those who did not.

Analyses were conducted using SPSS version 17. To assess the presence and degree of relationships between demographic, illness severity, cognitive and behavioural factors, and the two adjustment outcomes we performed Pearson's correlations (or ttests or ANOVA for the categorical data). Two separate hierarchical multiple regressions were conducted with GHQ and WSAS as the dependent variables in order to test the study's hypotheses. To determine whether the psychological factors accounted for variance in distress and functional impairment over and above illness severity and demographic variables these variables were entered on the first step, and the cognitive and behavioural variables were entered on the second step.

Due to the large number of analyses conducted we considered $\mathrm{p}<0.01$ as the level of significance when reporting results. However, we have highlighted both $p<.05$ and $p<.01$ in the tables for interest.

\section{Results}

\section{Participants}

The demographic and illness profiles of the participants are depicted in Table 1. The sample was $69.1 \%$ female, $75.5 \%$ White British with a mean age of 41.7. Most participants were married and highly educated and just over half were employed. Participants had been diagnosed with MS for a mean of 3.8 years, therefore the sample was relatively early in their disease trajectory. Most had relapsing-remitting 
MS. As a result of exclusion criteria of a TICS-M score of $<20$, no participants were considered to have substantial cognitive impairment (range $=20-35$, mean $=26.5$, $\mathrm{SD}=3.5$ ). The mean EDSS score was 4.85 indicating a combination of disability in a number of functional systems (e.g. problems with vision, co-ordination) and/or difficulty walking distances less than 500 metres

Table 1.

Participant demographic and illness characteristics $(n=94)$

Variable

$n(\%)$ or $\quad$ Range mean (SD)

\begin{tabular}{|c|c|c|}
\hline Age & 41.7 (9.6\%) & $21-66$ \\
\hline \multicolumn{3}{|l|}{ Gender } \\
\hline Female & 65 (69.1\%) & \\
\hline Male & $29(30.8 \%)$ & \\
\hline \multicolumn{3}{|l|}{ Ethnicity } \\
\hline White British & 71 (75.5\%) & \\
\hline Other White & 10 (10.6\%) & \\
\hline Other & 13 (13.9\%) & \\
\hline
\end{tabular}

\begin{tabular}{|c|c|c|}
\hline Education & & \\
\hline No formal & $1(1.1 \%)$ & \\
\hline GCSEs or A levels (or equivalent) & 45 (47.9\%) & \\
\hline Degree or postgraduate & $41(43.6 \%)$ & \\
\hline Other (e.g. vocational qualification) & 7 (7.4\%) & \\
\hline Marital status & & \\
\hline Married or living with partner & $54(57.4 \%)$ & \\
\hline Single & $28(29.8 \%)$ & \\
\hline Divorced or separated & $12(12.8 \%)$ & \\
\hline Employment status & & \\
\hline Employed $^{1}$ & $49(52.1 \%)$ & \\
\hline Not employed & 45 (47.9\%) & \\
\hline Time since diagnosis (years) & $3.8(2.8)$ & $0.8-10$ \\
\hline Type of MS & & \\
\hline Relapsing-remitting & $73(77.7 \%)$ & \\
\hline Primary progressive & $12(12.8 \%)$ & \\
\hline Secondary progressive & $9(9.6 \%)$ & \\
\hline EDSS Score & $5.0(1.2)$ & $0-7$ \\
\hline Relapses in last 12 months $^{2}$ & & \\
\hline None & 27 (32.9\%) & \\
\hline 1 to 3 & $44(53.7 \%)$ & \\
\hline More than 3 & $10(12.2 \%)$ & \\
\hline Missing & $1(1.2 \%)$ & \\
\hline Current relapse $^{2}$ & & \\
\hline Yes & 7 (8.5\%) & \\
\hline No & $74(90.2 \%)$ & \\
\hline Missing & $1(1.2 \%)$ & \\
\hline Cognitive Impairment (TICS-M score) & $26.5(3.5)$ & $20-35$ \\
\hline
\end{tabular}

1 includes full or part time, self-employed, reduced hours or sick leave

${ }^{2} n=82$ because patients with primary progressive MS do not experience relapses 


\section{Preliminary analyses}

Before testing the study's hypotheses regarding psychological factors associated with adjustment, relationships between key demographic and MS factors and the adjustment outcomes were investigated. Table 2 shows that age, gender, marital status, education and ethnicity were unrelated to either the WSAS or GHQ scores. Time since diagnosis, current and recent relapse status, and cognitive impairment were also unrelated to either adjustment outcome. MS type and EDSS were unrelated to GHQ scores. However people with progressive forms of MS had higher WSAS scores than those with relapsing remitting MS, indicating that progressive disease was associated with more functional impairment. EDSS had a medium strength positive relationship with WSAS, suggesting that increasing neurological disability was related to worse functional impairment. Since MS type and EDSS scores were related to adjustment outcomes, these were controlled for in later regression analyses.

Table 2

Relationships between demographic and MS factors and adjustment outcomes

\begin{tabular}{lll}
\hline & $\begin{array}{l}\text { Functional impairment } \\
\text { (WSAS) }\end{array}$ & Distress (GHQ) \\
\hline Demographic factors & $\begin{array}{l}\text { (1,91) }=-.073, p=.942 \\
\text { Gender }\end{array}$ & $\begin{array}{l}t(1,92)=1.163, p=.248 \\
r=.015\end{array}$ \\
Age & $F(3,89)=.062, p=.980$ & $F(3,90)=.573, p=.634$ \\
Marital status & $F(5,87)=1.362, p=.246$ & $F(5,88)=.857, p=.513$ \\
Education & $F(10,82)=.745, p=.680$ & $F(10,83)=.1 .042, p=.417$ \\
Ethnicity & & \\
Illness factors & $r=.123$ & $r=-.080$ \\
Time since diagnosis & $r=.475^{* *}$ & $r=.054$ \\
EDSS & $t(1,91)=-2.710, p=.008^{* *}$ & $t(1,92)=-1.428, p=.157$ \\
Type of MS & $t(1,91)=1.639, p=.105$ & $t(1,91)=.181, p=.857$ \\
Current relapse & $F(4,87)=.295, p=.880$ & $F(4,88)=.689, p=.601$ \\
Recent relapses & $r=.059$ & $r=-.089$ \\
Cognitive impairment (TICS-M) & & \\
\hline
\end{tabular}

${ }^{*} p<.05,{ }^{* *} p<.01$

\section{Correlates of adjustment}

Table 3 depicts the correlations between the cognitive and behavioural factors and the adjustment outcomes. WSAS scores were only associated with the cognitive and behavioural responses to symptoms and some of the illness perception items. Significant positive correlations were found between WSAS and the embarrassment avoidance subscale, the all-or-nothing and avoidance/rest subscales, and the behavioural responses total score. WSAS scores were also positively correlated with the illness perceptions identity and emotional response items. Thus, participants who endorsed unhelpful cognitive and behavioural responses to their symptoms and who held negative perceptions of their MS had higher functional impairment.

GHQ scores were correlated with most psychological variables measured in this study. Significant positive relationships were found between the GHQ and both unhelpful 
beliefs about the self and unhelpful beliefs about emotions. GHQ scores were also positively related to a number of cognitive responses to symptoms; catastrophising, symptom focusing and embarrassment avoidance subscales , as well as the cognitive responses total score. GHQ scores also correlated positively with the illness perceptions concern and coherence items. Thus, participants who endorsed more statements on these measures of maladaptive responses had higher distress scores. GHQ was also significantly negatively related to acceptance showing that acceptance of MS was related to less distress.

Bivariate correlation of WSAS and GHQ scores revealed that they were positively correlated but the effect size was small $(r=.215, p<0.05)$. Thus they appeared to be gauging different aspects of adjustment.

Table 3

Cognitive and behavioural correlates of adjustment outcomes

\begin{tabular}{|c|c|c|}
\hline & $\begin{array}{l}\text { Functional } \\
\text { Impairment } \\
\text { (WSAS) }\end{array}$ & $\begin{array}{l}\text { Distress } \\
\text { (GHQ) }\end{array}$ \\
\hline Unhelpful beliefs about emotions (BES) & .03 & $.33^{* *}$ \\
\hline Unhelpful beliefs about self (PVS) & .10 & $.51 * *$ \\
\hline Acceptance of MS (ACHC) & -.17 & $-.40 * *$ \\
\hline \multicolumn{3}{|l|}{ Symptom Responses (CBRSQ) } \\
\hline Total cognitive subscales & .23 & $.45^{* *}$ \\
\hline Fear avoidance & .17 & .11 \\
\hline Damage beliefs & .19 & $.21^{*}$ \\
\hline Catastrophising & .09 & $.38 * *$ \\
\hline Symptom focusing & $.21^{*}$ & $.38 * *$ \\
\hline Embarrassment avoidance & $.28 * *$ & $.44^{* *}$ \\
\hline Total behavioural subscales & $.44^{* *}$ & .09 \\
\hline All-or-nothing & $.32 * *$ & .16 \\
\hline Avoidance/rest & $.42 * *$ & .02 \\
\hline \multicolumn{3}{|l|}{ Illness perceptions (BIPQ) } \\
\hline Consequences & $\mathrm{n} / \mathrm{a}^{1}$ & $.25 *$ \\
\hline Timeline & -.12 & .15 \\
\hline Personal control & .07 & .20 \\
\hline Treatment control & .06 & -.00 \\
\hline Illness identity & $.48 * *$ & -.08 \\
\hline Concern & .20 & $.37 * *$ \\
\hline Coherence & -.12 & $.28 * *$ \\
\hline Emotional response & $.31^{* *}$ & $\mathrm{n} / \mathrm{a}^{1}$ \\
\hline
\end{tabular}

\footnotetext{
${ }^{1}$ Not calculated due to conceptual overlap between item and adjustment outcome in question

${ }^{*} p<.05,{ }^{* *} p<.01$
} 


\section{Hierarchical multiple regressions}

Two separate hierarchical multiple regressions were conducted with GHQ and WSAS as the dependent variables in order to determine the most important psychological predictors of the adjustment outcomes and whether these factors accounted for variance after the contribution of the relevant demographic and illness-related factors. Thus type of MS (relapsing-remitting vs. progressive forms) and EDSS were entered onto the first step. The second step included the cognitive and behavioural factors that were significantly correlated with adjustment outcomes at $\mathrm{p}<.01$. Total scores for each variable were used except where some subscales were not significant correlates. In these cases the significant subscales were entered.

Table 4

Hierarchical multiple regression of the demographic, illness and cognitive and behavioural factors on functional impairment (WSAS)

\begin{tabular}{llll}
\hline Step and variable & $B$ & $S E B$ & $\beta$ \\
\hline 1. Control variables & & & \\
$\quad$ Constant & -1.598 & 3.379 & - \\
$\quad$ EDSS & 3.012 & .689 & $.438^{* *}$ \\
$\quad$ Type of MS & 1.982 & 1.952 & .102 \\
& & & \\
$R^{2}=.237$ & & & \\
$F=13.835(2,89), p<.001$ & & & \\
\hline
\end{tabular}

2. Cognitive \& Behavioural variables

$\begin{array}{llll}\text { Constant } & -11.942 & 3.861 & - \\ \text { EDSS } & 1.815 & .647 & .246^{* *} \\ \text { Type of MS } & 2.727 & 1.751 & .140 \\ \text { Symptom responses (CBRSQ)-behavioural subscale } & .312 & .098 & .289^{* *} \\ \text { Symptom responses (CBRSQ)-cognitive subscale } & .058 & .066 & .085 \\ \text { Illness perceptions (BIPQ)- illness identity item } & .953 & .347 & .246^{* *} \\ \text { Illness perceptions (BIPQ)- emotional response item } & .382 & .342 & .105\end{array}$

$R^{2}$ change $=.226$

$F=12.211(6,85), p<.001$

Total $R^{2}=.463$, Adjusted $R^{2}=.425$

${ }^{*} p<.05,{ }^{* *} p<.01$

The results for WSAS are shown in Table 4. EDSS and MS type accounted for a significant $23.7 \%$ of the variance, with EDSS emerging as the significant correlate on step 1. At step 2, however, patients' symptom responses and illness perceptions accounted for a further $22.6 \%$ of the variance in WSAS. The correlates significant at $\mathrm{p}<.01$ were behavioural responses to symptoms (all-or-nothing patterns of activity, or 
excessive avoidance and rest) and the illness perception identity item (attributing a large number of everyday symptoms to MS). Overall the model accounted for $46.3 \%$ of the variance in WSAS scores.

Table 5

Hierarchical multiple regression of the demographic, illness and cognitive and behavioural factors on distress (GHQ)

\begin{tabular}{|c|c|c|c|}
\hline Step and variable & $B$ & SE B & $\beta$ \\
\hline \multicolumn{4}{|l|}{ 1. Control variables } \\
\hline Constant & 14.689 & 2.887 & - \\
\hline EDSS & -.005 & .585 & -.001 \\
\hline Type of MS & 2.197 & 1.670 & .148 \\
\hline $\begin{array}{l}R^{2}=.022 \\
F=.999(2,90), p=.372\end{array}$ & & & \\
\hline
\end{tabular}

2. Cognitive \& Behavioural variables

Constant

EDSS

$\begin{array}{lll}-.238 & 6.092 & - \\ .500 & .507 & .096 \\ 1.524 & 1.425 & .103 \\ .403 & .136 & .348 * * \\ .115 & .079 & .134 \\ -.056 & .100 & -.067 \\ .053 & .058 & .107 \\ .117 & .311 & .066 \\ .530 & .257 & .191^{*}\end{array}$

Type of MS

Unhelpful beliefs about self (PVS)

Unhelpful beliefs about emotions (BES)

Acceptance of MS (ACHC)

Symptom responses (CBRSQ)- cognitive subscale

Illness perceptions (BIPQ)- concern item

$.191^{*}$

$R^{2}$ change $=.371$

$F=6.798(8,84), p<.001$

Total $R^{2}=.393$, Adjusted $R^{2}=.335$

${ }^{*} p<.05,{ }^{* *} p<.01$

Table 5 shows the hierarchical multiple regression results for GHQ scores. MS type and EDSS accounted for a non-significant 2.2\% of the variance in GHQ scores.

However, the cognitive and behavioural factors accounted for a significant $37.1 \%$ of the variance. Examination of the beta weights suggested that the strongest correlate of GHQ score was unhelpful beliefs about the self (e.g. need for approval, perfectionism). Overall the model accounted for $39.3 \%$ of the variance in GHQ scores. 


\section{Discussion}

The results of this study were broadly in line with our expectations. Distress was associated with both generic and illness-specific unhelpful beliefs and behaviours. However, functional impairment was only related to illness-specific thoughts and behaviours. The results demonstrate the importance of recognising various domains of adjustment in MS and considering different predictors for explaining different aspects Furthermore, cognitive and behavioural factors were important above and beyond the contribution of demographic and illness factors which played a relatively small role in adjustment. Disease factors were not strong correlates of distress, although neurological-related disability (EDSS score) was a significant correlate of functional impairment.

For functional impairment, behavioural responses to symptoms such as all-or-nothing patterns of activity and excessive rest or avoidance were key correlates. The importance of behavioural responses concurs with the only existing study that considered this variable. That study found that avoidance and resting in response to symptoms was a key predictor of poorer functional impairment (16). We found that out of the range of illness perceptions only illness identity (perceiving that lots of symptoms were associated with MS) was a strong predictor of illness impact. This finding differs from an earlier study where a range of illness perceptions consistently predicted significant variance in adjustment outcomes (15). In that study, perceiving severe consequences of MS was the main predictor of role dysfunction (a similar concept to functional impairment), whereas illness identity was not a significant predictor (15). Differences between our findings and previous findings may be explained by the fact that we excluded the consequences BIPQ dimension from analyses of predictors of functional impairment as we deemed there to be a considerable overlap in the concepts they were measuring. Furthermore, the measure of illness perceptions was slightly different in the two studies (this study used the BIPQ (40) whereas the other study used the IPQ-R (42). As far as we are aware this is the first study to examine unhelpful beliefs about the self, beliefs about emotions, and acceptance in relation to impact of MS on life roles. The findings that these factors were unrelated to functional impairment corresponded to our hypotheses.

In contrast, for distress, unhelpful beliefs about the self such as feeling inferior, needing others' approval, and feeling like a failure if goals are not achieved emerged as the strongest correlate. This is in line with our proposition that it is more generic, rather than illness-specific cognitions and behaviours, that are particularly relevant for explaining distress. This also concurs with previous research which linked maladaptive thinking styles to depression in MS (21;23;24). One explanation of this finding is that these thinking styles develop through early experiences and are present prior to MS development. However, these prove particularly unhelpful in the context of the major stressors that MS produces and promote poor adjustment. The finding that unhelpful beliefs about emotions and lack of acceptance of MS were correlated with distress is also in line with expectations and some existing literature $(18 ; 30)$. Interestingly these were not important predictors of distress in the regression analysis when considered alongside unhelpful beliefs about the self. This suggests that addressing dysfunctional assumptions about the self may be key to moderating levels 
of distress in MS. Assisting patients to accept MS and to express negative emotions may also lessen distress but appears to be less important.

The non-significant influence of disease variables on more emotional adjustment outcomes correspond to many previous studies (e.g. 15;43) and fit well with a cognitive behavioural model of adjustment to MS (14). Thus, whilst disease factors are important triggers for adjustment difficulties, an individual's cognitions and behaviours contribute significantly to psychological adjustment. In this study the importance of cognitive and behavioural factors for explaining distress and functional impairment was substantial. However, the combinations of variables we investigated explained only $22.6 \%$ and $37,1 \%$ of the variance in functional impairment and distress respectively. Therefore, other variables are also likely to be relevant to understanding adjustment.

Knowledge about the correlates of adjustment is important for identifying who may be vulnerable to adjustment difficulties in the future. For example, it may be possible to target adjustment-related interventions at patients who are starting to develop avoidant responses to their MS symptoms (e.g. resting excessively in the hope of reducing symptoms) to limit future functional impairment.

The relevance of cognitive and behavioural variables in adjustment above and beyond MS severity variables gives cause for hope for interventions to assist adjustment. Whereas the scope for modifying the course and severity of MS remains modest for many PwMS, cognitions and behaviours are potentially modifiable. Approaches using cognitive behaviour therapy (CBT) may be helpful in this regard. A recent Cochrane review concluded that CBT is effective for treating depression in people with MS (44). However, it is yet unclear whether CBT is an efficacious intervention for promoting adjustment in people without diagnosed mental health problems but with other, sub clinical adjustment difficulties. Nonetheless, this study provides some preliminary suggestion as to potential areas of focus within CBT-based interventions. Depending on the aim of the intervention - reducing distress, improving functioning, or bothclinicians may opt to select the strongest correlates of that adjustment outcome as a focus for change. For example, an intervention primarily aiming to reduce distress in clients may usefully tackle unhelpful cognitions (e.g. around perfectionism and need for approval) by using thought diaries, and training in developing alternative and more helpful thoughts. On the other hand, an intervention aiming to help people engage in a more active social life and contribution to home life may concentrate on symptom-related behaviours and thoughts by helping the client to develop more consistent patterns of activity and rest through planning, monitoring and graded activity, and to identify and modify unhelpful and inaccurate beliefs about MS and symptoms.

A major limitation of this study is that due to its cross-sectional design causal relationships cannot be inferred. Longitudinal studies are required to explore relationships further. Intervention studies examining changes in these cognitive and behavioural factors as mechanisms of any treatment effects would add weight to the argument that these play a causal role in adjustment outcomes. 
Another limitation is that because the study was nested within a therapy trial, with strict eligibility criteria and substantial time commitment required to participate, the sample may not be representative of PwMS. Furthermore, whilst we specifically aimed to sample people in the early stages of MS, replication in a larger, broader sample, including patients with higher levels of disability and longer illness duration would be valuable.

Finally, the lack of influence of MS type, current and recent relapse status, and disability on adjustment may be related to the use of self-report questions for determining these factors. A broader examination of these factors, by conducting neurologist examinations on entry to the trial may have revealed more but was beyond the scope of the study.

Unhelpful thoughts and behaviours, both MS specific and more general are important in understanding variations in adjustment outcomes in PwMS. Though more research is needed, attempts to address these factors within psychological interventions could have a beneficial effect on people’s adjustment to this difficult disease.

\section{Acknowledgements}

This study was funded by a UK MS Society project grant with Rona Moss-Morris as the lead investigator. Trudie Chalder is supported by NIHR Biomedical Research Centre, South London and Maudsley NHS Foundation Trust / Institute of Psychiatry King's College London. Thank you to all who participated. Thank you also to our colleagues at the NHS MS Services at Wessex and King's College Hospital for their assistance with recruitment: Alan Turner, Chris Halfpenny, Sheila Chartres, Jane Ware, Jane Cameron, Hazel Daniel, Stephanie Heath, Joan Regan, Pauline Shaw, Fiona Barnes and Sally Jones.

\section{Reference List}

(1) National MS Society. Who gets MS? http://www.nationalmssociety.org/aboutmultiple-sclerosis/who-gets-ms/index.aspx . 2009. 18-2-2009.

Ref Type: Online Source

(2) Goodkin D. Interferon beta therapy for multiple sclerosis. The Lancet 1998;352:1486-7.

(3) Goodin DS. Disease-modifying therapy in multiple sclerosis: Update and clinical implications. Neurology 2008 Dec 9;71(24_suppl_3):8-13.

(4) Rudick RA. Disease-modifying drugs for relapsing-remitting multiple sclerosis and future directions for multiple sclerosis therapeutics. Arch Neurol 1999 Sep 1;56(9):1079-84.

(5) Arnett P, Barwick F, Beeney J. Depression in multiple sclerosis: Review and theoretical proposal. Journal of the International Neuropsychological Society 2008;14(05):691-724. 
(6) Janssens A, van Dorn P, de Boer J, van der Meche F, Passchier J, Hintzen R. Impact of recently diagnosed multiple sclerosis on quality of life, anxiety, depression and distress of patients and partners. Acta Neurologica Scandinavica 2003;108(6):389-95.

(7) Zorzon M, de Masi R, Nasuelli D, Ukmar M, Pozzi Mucelli R, Cazzato G, et al. Depression and anxiety in Multiple Sclerosis: a clinical and MRI study in 95 subjects. Journal of Neurology 2001;248(5):1432-59.

(8) Hakim EA, Bakheit AMO, Bryant TN, Roberts MWH, McIntosh-Michaelis SA, Spackman AJ, et al. The social impact of multiple sclerosis-a study of 305 patients and their relatives. Disability and Rehabilitation 2000;22(6):288-93.

(9) Mohr D, Dick L, Russo D, Pinn J, Boudewyn A, Goodkin D, et al. The psychosocial impact of multiple sclerosis: Exploring the patient's perspective. Health Psychology 1999;18(4):376-82.

(10) Benito-Leon J, Morales MJ, Rivera-Navarro J, Mitchell A. A review about the impact of multiple sclerosis on health-related quality of life. Disability and Rehabilitation 2003;25:1291-304.

(11) Chwastiak L, Ehde DM, Gibbons LE, Sullivan M, Bowen JD, Kraft GH. Depressive symptoms and severity of illness in multiple sclerosis: Epidemiologic study of a large community sample. Am J Psychiatry 2002 Nov 1;159(11):1862-8.

(12) McIvor G, Rikland M, Reznikoff M. Depression in multiple sclerosis as a function of length and severity of illness, age, remissions, and perceived social support. Journal of Clinical Psychology 1984;40:1028-33.

(13) Kurtzke J. Rating neurological impairment in multiple sclerosis: an expanded disability status scale (EDSS). Neurology 1983;33:1444-52.

(14) Dennison L, Moss-Morris R, Chalder T. A review of psychological correlates of adjustment in patients with multiple sclerosis. Clinical Psychology Review 2009;29:141-53.

(15) Jopson NM, Moss-Morris R. The role of illness severity and illness representations in adjusting to multiple sclerosis. Journal of Psychosomatic Research 2003;54(6):503-11.

(16) Skerrett TN, Moss-Morris R. Fatigue and social impairment in multiple sclerosis: The role of patients' cognitive and behavioral responses to their symptoms. Journal of Psychosomatic Research 2006;61:587-93.

(17) Dupont S. Sexual function and ways of coping in patients with multiple sclerosis and their partners. Sexual and Marital Therapy 1996;11(4):359-72.

(18) Harrison T, Stuifbergen A, Adachi E, Becker H. Marriage, impairment, and acceptance in persons with multiple sclerosis. Western Journal of Nursing Research 2004 Apr;26(3):266-85. 
(19) Telford K, Kralik D, Koch T. Acceptance and denial: implications for people adapting to chronic illness: literature review. Journal of advanced nursing 2006;55(4):457-64.

(20) Bruce JM, Polen D, Arnett PA. Pain and affective memory biases interact to predict depressive symptoms in multiple sclerosis. Multiple Sclerosis 2007 Jan;13(1):58-66.

(21) Kneebone II, Dunmore EC, Evans E. Symptoms of depression in older adults with multiple sclerosis (MS): comparison with a matched sample of younger adults. Aging \& Mental Health 2003 May;7(3):182-5.

(22) Kneebone II, Dunmore E. Attributional style and symptoms of depression in persons with multiple sclerosis. International Journal of Behavioral Medicine 2004;11(2):110-5.

(23) Shnek ZM, Foley FW, LaRocca NG, Smith CR, Halper J. Psychological predictors of depression in multiple sclerosis. Journal of Neurologic Rehabilitation 1995;9(1):15-23.

(24) Shnek ZM, Foley FW, LaRocca NG, Gordon WA, DeLuca J, Schwartzman HG, et al. Helplessness, self-efficacy, cognitive distortions, and depression in multiple sclerosis and spinal cord injury. Annals of Behavioral Medicine 1997;19(3):287-94.

(25) Dennison L, Yardley L, Devereux A, Moss-Morris R. Experiences of adjusting to early stage MS; the patient's perspective. In preparation 2009.

(26) Ali A, Toner B, Stuckless N, Gallop R, Diamant N, Gould M, et al. Emotional abuse, self-blame, and self-silencing in women with irritable bowel syndrome. Psychosomatic Medicine 2000;62:76-82.

(27) Cramer KGM, Langlois M. Self-silencing and depression in women and men: comparative structural equation models. Personality and Individual Differences 2005;39:581-92.

(28) Jack DC. Silencing the self: Women and depression. Cambridge: Harvard University Press; 1991.

(29) Surawy C, Hackmann A, Hawton K, Sharpe M. Chronic fatigue syndrome: a cognitive approach. Behaviour Research and Therapy 1995;33(5):535-44.

(30) Rimes K, Chalder T. The beliefs about emotions scale (BES): validity, reliability and sensitivity to change. Journal of Psychosomatic Research 2009; IN PRESS.

(31) Moss-Morris R, Dennison L, Yardley L, Landau S, Roche S, McCrone P, et al. Protocol for the saMS trial (supportive adjustment for multiple sclerosis): A randomized controlled trial comparing cognitive behavioural therapy to supportive listening for adjustment to multiple sclerosis. BMC Neurology 2009. 
(32) Brandt J, Welsh K, Breitner J, Folstein M, Helms M, Christian J. Hereditary influences on cognitive functioning in older men; a study of 4000 pairs. Arch Neurol 1993;50:599-603.

(33) Bowen J, Gibbons L, Gianas A, Kraft G. Self-administered Expanded Disability Status Scale with functional system scores correlates well with physician-administered test. Multiple Sclerosis 2001;7:201-6.

(34) Mundt J, Marks I, Shear K, Greist J. The Work and Social Adjustment Scale: a simple measure of impairment in functioning. British Journal of Psychiatry 2002;180:461-4.

(35) van Kessel K, Moss-Morris R, Willoughby E, Chalder T, Johnson M, Robinson E. A randomized controlled trial of cognitive behavior therapy for multiple sclerosis fatigue. Psychosomatic Medicine 2008;70:205-13.

(36) Goldberg D. General Health Questionnaire (GHQ-12). Windsor: NFERNELSON; 1992.

(37) Hobart J, Riazi A, Lamping D, Fitzpatrick R, Thompson A. How responsive is the Multiple Sclerosis Impact Scale (MSIS-29)? A comparison with some other self report scales. Journal of Neurology Neurosurgery and Psychiatry 2006;76:-1539.

(38) Sinclair VG, Wallston KA. The development and validation of the Psychological Vulnerability Scale. Cognitive Therapy and Research 1999;23(2):119-29.

(39) Stuifbergen A. Conceptualization and development of the Acceptance of Chronic Health Conditions Scale. Issues in Mental Health Nursing 2008;29(2):101-14.

(40) Broadbent E, Petrie KJ, Main J, Weinman J. The Brief Illness Perception Questionnaire. Journal of Psychosomatic Research 2006;60(6):631-7.

(41) Moss-Morris R, Chalder T, Skerrett T, Baldwin M. The Cognitive and Behavioural Responses to Symptoms Questionnaire: measuring cognitive and behavioural aspects of symptom interpretation. In preparation 2009.

(42) Moss-Morris R, Weinman J, Petrie K, Horne R, Cameron L. The revised illness perception questionnaire (IPQ-R). Psychology and Health 2002;17:1-6.

(43) McIvor G. Depression in Multiple Sclerosis as a Function of Length and Severity of Illness, Age, Remissions, and Social Support. Journal of Clinical Psychology 1984;40(4):1028-33.

(44) Thomas PW, Thomas S, Hiller C, Galvin K, Baker R. Psychological interventions for multiple sclerosis. Cochrane Database of Systematic Reviews 2006;1(CD004431). 